12

\title{
Новые наноразмерные люминофоры, полученные испарением силикатов и германатов Р3Э
}

\author{
() М.Г. Зуев ${ }^{1}$, В.Г. Ильвес ${ }^{2}$, С.Ю. Соковнин ${ }^{2}$, А.А. Васин ${ }^{1}$, И.В. Бакланова ${ }^{1}$ \\ ${ }^{1}$ Институт химии твердого тела УрО РАН, \\ Екатеринбург, Россия \\ ${ }^{2}$ Институт электрофизики УрО РАН, \\ Екатеринбург, Россия \\ E-mail: Zuev@ihim.uran.ru
}

Методом испарения импульсным электронным пучком мишеней из поликристаллических фосфоров состава $\mathrm{Ca}_{2} M_{8}\left(\mathrm{SiO}_{4}\right)_{6} \mathrm{O}_{2}: \mathrm{Eu}(M=\mathrm{Y}, \mathrm{Gd})$ и $\mathrm{Ca}_{2} \mathrm{La}_{8}\left(\mathrm{GeO}_{4}\right)_{6} \mathrm{O}_{2}: \mathrm{Eu}$ со структурой оксиапатита впервые получены нанофосфоры в аморфном состоянии. Обнаружено восстановление ионов $\mathrm{Eu}^{3+} \rightarrow \mathrm{Eu}^{2+}$ в электронном пучке. Обнаружена модификация спектров комбинационного рассеяния света (КР) образцов при уменьшении частиц от объемного до наноразмерного состояния. Рассмотрено изменение ширины запрещенной зоны $E_{g}$ образцов при переходе от объемного порошка к НП. Изучены спектрально-люминесцентные характеристики образцов в поликристаллическом и наноаморфном состояниях. Показано, что при переходе к нанообразцам поле лигандов вокруг $\mathrm{Eu}^{2+}$ изменяется. Это может быть обусловлено нарушением трансляционной симметрии в НП. Ослабляется связь $4 f$ - и $5 d$-электронов. Возникает вырождение уровня ${ }^{2} e_{g}$. Предположительно обнаружено восстановление ионов $\mathrm{Eu}^{3+} \rightarrow \mathrm{Eu}^{2+}$ в электронном пучке за счет разрыва связи $\mathrm{Si}(\mathrm{Ge})-\mathrm{O}$ в процессе испарения образцов и захвата высвободившегося электрона ионами $\mathrm{Eu}^{3+}$.

Авторы признательны УрО РАН за финансовую поддержку (проект № 18-10-3-32) и А.М. Мурзакаеву за микроскопический анализ НП.

DOI: 10.21883/FTT.2019.05.47610.34F

\section{1. Введение}

Исследование влияния уменьшения частиц от объемного до наноразмерного состояния на формирование свойств люминофоров является актуальным. В ряде работ рассматривается влияние наноразмерного состояния образцов на интенсивность свечения ионов РЗЭ, в частности, $\mathrm{Tb}^{3+}, \mathrm{Eu}^{3+}[1,2]$. В статье [2], например, показано, что интенсивность свечения ионов $\mathrm{Eu}^{3+}$ в объемных люминофорах $\mathrm{YBO}_{3}: \mathrm{Eu}^{3+}$ выше, чем в соответствующих нанолюминофорах, синтезированных методом соосаждения. В работе [3], наоборот, интенсивность свечения $\mathrm{Eu}^{3+}$ выше в аморфных нанолюминофорах, чем в объемных образцах $\mathrm{Sr}_{2} \mathrm{Gd}_{8}\left(\mathrm{SiO}_{4}\right)_{6} \mathrm{O}_{2}: \mathrm{Eu}$. Нанолюминофоры получены испарением электронным пучком (ИЭП) мишеней из указанных силикатов. Вызывает интерес изучение зависимости спектрально-люминесцентных характеристик нанолюминофоров от способа их получения.

Силикатные и германатные матрицы, содержащие ионы РЗЭ, известны как эффективные люминофоры, перспективные, например, для конструирования LED устройств [8]. Имеется ряд статей, посвященным силикатным и германатным нано- и объемным люминофорам, полученным различными способами и содержащим ионы $\mathrm{Eu}^{3+}$ и $\mathrm{Eu}^{2+}[9-13,14]$. Нанолюминофоры во многих случаях получают с помощью золь-гель процессов.

В работе [3] методом ИЭП из поликристаллических фосфоров $\mathrm{Sr}_{2} \mathrm{Gd}_{8}\left(\mathrm{SiO}_{4}\right)_{6} \mathrm{O}_{2}: \mathrm{Eu}$ со структурой оксиапатита впервые получены нанофосфоры в аморфном состоянии. Методами дифференциальной сканирующей калориметрии (DSC) и термогравиметрии (TG) изучены процессы кристаллизации аморфного образца. Изучены спектрально-люминесцентные характеристики фосфоров $\mathrm{Sr}_{2} \mathrm{Gd}_{6.4} \mathrm{Eu}_{1.6} \mathrm{Si}_{6} \mathrm{O}_{26-\delta}$ в поликристаллическом и наноаморфном состояниях. Обнаружена модификация КР-спектров при уменьшении частиц от объемного до наноразмерного состояния. Наряду с изолированными группами $\mathrm{SiO}_{4}$ в наноаморфных образцах возникает небольшая конденсация тетраэдров $\mathrm{SiO}_{4}$, аналогичная эффекту полимеризации в быстрозакаленных силикатных расплавах. В книге [15] детально описан метод ИЭП для получения люминесцентных материалов, в том числе и силикатных.

Цель настоящего исследования - разработать способ получения новых сложнооксидных силикатных и германатных нанолюминофоров, содержащих ионы европия, рассмотреть механизм восстановления ионов $\mathrm{Eu}^{3+} \rightarrow \mathrm{Eu}^{2+}$ в процессе испарения поликристаллических фосфоров методом ИЭП, определить спектрально-люминесцентные характеристики полученных фосфоров. В работе методом ИЭП поликристаллических фосфоров составов $\mathrm{Ca}_{2} \mathrm{Y}(\mathrm{Gd})_{8}\left(\mathrm{SiO}_{4}\right)_{6} \mathrm{O}_{2}: \mathrm{Eu}$ и $\mathrm{Ca}_{2} \mathrm{La}_{8}\left(\mathrm{GeO}_{4}\right)_{6} \mathrm{O}_{2}: \mathrm{Eu}$ впервые получены нанофосфоры в аморфном состоянии. Методами DSC и TG изучены процессы кристаллизации аморфных нанопорошков. Обнаружена модификация КР-спектра и изменение ширины запрещенной зоны образцов при уменьшении частиц от объемного до наноразмерного состояния. Изучены спектрально-люминесцентные характеристики в поликристаллическом и наноаморфном состояниях. Установ- 
лено, что при переходе в наноаморфное состояние люминофоры изменяют цвет фотолюминесценции с краснооранжевого $\left(\mathrm{Eu}^{3+}\right)$ на синий $\left(\mathrm{Eu}^{2+}\right)$ и белый.

\section{2. Методика эксперимента}

Синтез поликристаллических образцов осуществляли обжигом на воздухе смеси компонентов. Препаративные методы синтеза аналогичны методам, описанным в работе [3,16]. Использовали две стадии синтеза силикатов. На первой стадии в качестве исходных компонентов использовали реактивы $\mathrm{CaCO}_{3}, \mathrm{Y}_{2} \mathrm{O}_{3}$, $\mathrm{Gd}_{2} \mathrm{O}_{3}, \mathrm{Eu}_{2} \mathrm{O}_{3}, \mathrm{SiO}_{2}$ с содержанием основного вещества не менее $99.99 \%$ и синтезировали соединения $\mathrm{Ca}_{2} \mathrm{Gd}_{8}\left(\mathrm{SiO}_{4}\right)_{6} \mathrm{O}_{2}, \mathrm{Ca}_{2} \mathrm{Y}_{8}\left(\mathrm{SiO}_{4}\right)_{6} \mathrm{O}_{2}$ и $\mathrm{Ca}_{2} \mathrm{Eu}_{8}\left(\mathrm{SiO}_{4}\right)_{6} \mathrm{O}_{2}$. Стехиометрические количества исходных компонентов были смешаны в агатовой ступке. Затем порошки были и обожжены на воздухе в температурном интервале $1000-1400^{\circ} \mathrm{C}$. Время обжига составило $\sim 80 \mathrm{~h}$. На второй стадии синтеза вводили европий. С этой целью в качестве исходных компонентов брали указанные соединения в стехиометрическом соотношении для получения твердых растворов состава $\mathrm{Ca}_{2} \mathrm{Gd}_{8(1-x)} \mathrm{Eu}_{8 x}\left(\mathrm{SiO}_{4}\right)_{6} \mathrm{O}_{2}$ и $\left.\mathrm{Ca}_{2} \mathrm{Y}_{8(1-x)} \mathrm{Eu}_{8 x} \mathrm{SiO}_{4}\right)_{6} \mathrm{O}_{2}$. Вещества тщательно перемешивали и обжигали на воздухе при температуре $1450^{\circ} \mathrm{C}$ в течение $\sim 20 \mathrm{~h}$ с промежуточным перетиранием порошков. После чего печь произвольно охлаждалась с выключенными нагревателями.

Для синтеза $\mathrm{Ca}_{2} \mathrm{La}_{8(1-x)} \mathrm{Eu}_{8 x}\left(\mathrm{GeO}_{4}\right)_{6} \mathrm{O}_{2}$ в качестве исходных компонентов были использованы $\mathrm{CaCO}_{3}, \mathrm{La}_{2} \mathrm{O}_{3}$, $\mathrm{Eu}_{2} \mathrm{O}_{3}$ и $\mathrm{GeO}_{2}$. Соединения $\mathrm{CaCO}_{3}, \mathrm{La}_{2} \mathrm{O}_{3}$ и $\mathrm{Eu}_{2} \mathrm{O}_{3}$, взятые в стехиометрическом соотношении, растворяли в концентрированном $\mathrm{HNO}_{3}$. Раствор $\mathrm{GeO}_{2}$ был приготовлен в избытке $\mathrm{NH}_{4} \mathrm{OH}$ при температуре $80^{\circ} \mathrm{C}$ в течение $40 \mathrm{~min}$. После того, как раствор содержащий $\mathrm{Ca}\left(\mathrm{NO}_{3}\right)_{2}$, $\mathrm{La}\left(\mathrm{NO}_{3}\right)_{3}, \mathrm{Eu}\left(\mathrm{NO}_{3}\right)_{3}$ был перенесен в аммиачный раствор оксида германия, полученная смесь упаривалась при температуре $120^{\circ} \mathrm{C}$ до образования белого осадка.

Полученный материал затем подвергали термической обработке в несколько стадий: первая стадия включала нагрев от 200 до $800^{\circ} \mathrm{C}$ с шагом $100^{\circ} \mathrm{C} / \mathrm{h}$, вторая включала спекание при $1000^{\circ} \mathrm{C}$ в течение $14 \mathrm{~h}$, а третья стадия включала отжиг при $1200^{\circ} \mathrm{C}$ в течение $28 \mathrm{~h}$.

Полноту синтеза проверяли рентгенофазовым анализом (РФА). РФА проводили с помощью дифрактометра Shimadzu XRD-7000 ( $\mathrm{CuK}_{\alpha}$-излучение) с использованием картотеки ICDD. Сканирование осуществляли при комнатной температуре. Дифрактограммы полученных образцов, снятых в интервале углов $2 \theta=5-70^{\circ}$, обработаны по методу полнопрофильного анализа Ритвельда.

Нанопорошки (НП) получали испарением керамических мишеней в вакууме и атмосфере аргона (давление 10-20 Ра) с помощью ИЭП на установке НАНОБИМ2 [15]. Мишени в виде дисков, диаметром 20-30 mm, высотой до 20 мм, изготовленных из объемных люминофоров, получали при обжиге дисков при $1400^{\circ} \mathrm{C}$ в течение $40 \mathrm{~h}$ ). Энергия электронов составляла $40 \mathrm{keV}$, энергия импульса электронного пучка $1.8 \mathrm{~J}$, длительность импульса $100 \mu \mathrm{s}$, частота импульсов $100-200 \mathrm{~Hz}$. Время испарения мишени - 40-60 min. Скорость вращения мишени составляла $8.3 \mathrm{rpm}$. НП осаждали на неохлаждаемые стеклянные подложки, размещенные вокруг мишени. Сбор НП со стеклянных подложек выполняли с помощью фольги из титана. Удельную поверхность порошков $\left(S_{\text {уд }}\right)$ определяли методом Брунауэра-Эммета-Тейлора (BET) [17] на установке Micromeritics TriStar 3000.

Микроскопический анализ НП проводили на просвечивающем электронном микроскопе JEOL JEM 2100. КР-спектры записаны на спектрометре Renishaw $\left(\Delta v=1000 \mathrm{~cm}^{-1}\right)$ с использованием аргонового лазера $(\lambda=514.5 \mathrm{~nm})$.

Для экспериментальной оценки ширины запрещенной зоны записаны спектры поглощения. Спектры поглощения записаны на приборе UV-2401 PC фирмы Shimadzu. Край поглощения определяли экстраполяцией кривой линейной зависимости коэффициента поглощения до пересечения ее с осью абсцисс (длин волн). Точка пересечения определяет край поглощения.

Спектры фотолюминесценции и возбуждения записаны соответственно на спектрометрах МДР-204 (дейтериевая лампа, ФЭУ R928 фирмы Hamamatsu) и Cary Eclipse Fluorescence Spectrophotometer (импульсная ксеноновая лампа (частота $80 \mathrm{~Hz}$, длительность импульса $\sim 2 \mu \mathrm{s}$, пиковая мощность $75 \mathrm{~kW})$.

\section{3. Результаты и обсуждение}

На рис. 1 представлена характерные дифрактограммы синтезированных твердых растворов $\mathrm{Ca}_{2} M_{6.4} \mathrm{Eu}_{1.6}\left(\mathrm{SiO}_{4}\right)_{6} \mathrm{O}_{2} \quad(M=\mathrm{Y}, \mathrm{Gd}) \quad$ и $\mathrm{Ca}_{2} \mathrm{La}_{6.4} \mathrm{Eu}_{1.6}\left(\mathrm{GeO}_{4}\right)_{6} \mathrm{O}_{2}$. Твердые растворы относятся к структурному типу оксиапатита [18]. Выбор этих составов обусловлен тем, что в ряду люминофоров $\mathrm{Ca}_{2} M_{8(1-x)} \mathrm{Eu}_{8 x}\left(\mathrm{SiO}_{4}\right)_{6} \mathrm{O}_{2} \quad$ и $\mathrm{Ca}_{2} \mathrm{La}_{8(1-x)} \mathrm{Eu}_{8 x}\left(\mathrm{GeO}_{4}\right)_{6} \mathrm{O}_{2}$ твердые растворы при $x=0.2$ имеют максимальную интегральную интенсивность люминесценции ионов $\mathrm{Eu}^{3+}$. При $x>0.2$ наблюдается концентрационное тушение люминесценции $\mathrm{Eu}^{3+}$. После обработки методом Ритвельда получены следующие данные (таблица). Также в таблице даны величины плотностей соединений. Полученные на основе указанных твердых растворов НП - рентгеноаморфны.

Электронная микроскопия НП, полученного на основе $\mathrm{Ca}_{2} \mathrm{Y}_{6.4} \mathrm{Eu}_{1.6} \mathrm{Si}_{6} \mathrm{O}_{26-\delta}$, где $\delta$ - кислородная нестехиометрия, показала, что наночастицы достаточно сильно агломерированы, имеют неправильную форму с тенденцией к образованию квазисферических частиц. Кислородная нестехиометрия возникает из-за образования ионов $\mathrm{Eu}^{2+}$ (показано ниже). На (рис. 2,a) показаны частицы нанообразца. TEM HR снимки показали, что НП состоит исключительно из аморфных частиц. 
Характеристики объемных образцов

\begin{tabular}{c|c|c|c|c|c}
\hline $\begin{array}{c}\text { Образец, } \\
\text { № }\end{array}$ & Состав & $a, \AA$ & $b, \AA$ & $V, \AA^{3}$ & $\begin{array}{c}\text { Плотность, } \\
\mathrm{g} / \mathrm{cm}^{3}[16,19]\end{array}$ \\
\hline 1 & $\mathrm{Ca}_{2} \mathrm{Y}_{6.4} \mathrm{Eu}_{1.6}\left(\mathrm{SiO}_{4}\right)_{6} \mathrm{O}_{2}$ & 9.3472 & 6.7828 & 513.220 & $\sim 4.5$ \\
2 & $\mathrm{Ca}_{2} \mathrm{Gd}_{6.4} \mathrm{Eu}_{1.6}\left(\mathrm{SiO}_{4}\right)_{6} \mathrm{O}_{2}$ & 9.4178 & 6.8969 & 529.76 & $\sim 6$ \\
3 & $\mathrm{Ca}_{2} \mathrm{La}_{6.4} \mathrm{Eu}_{1.6}\left(\mathrm{GeO}_{4}\right)_{6} \mathrm{O}_{2}$ & 9.8213 & 7.1656 & 598.58 & $\sim 5.7$
\end{tabular}

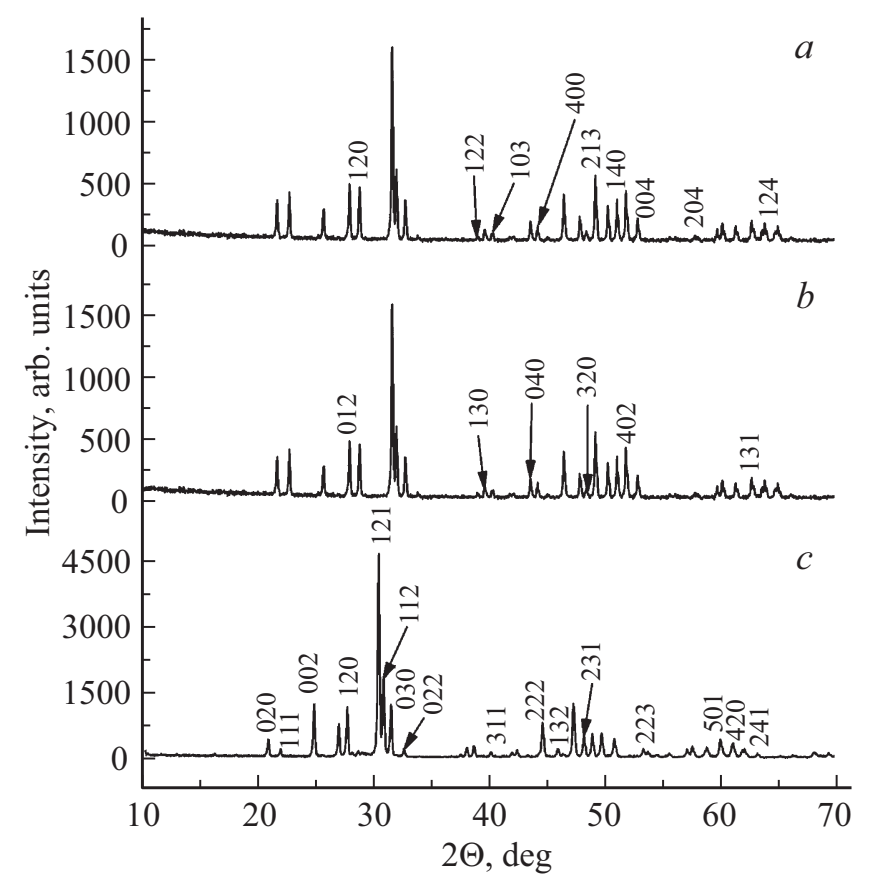

Рис. 1. Дифрактограммы соединений: $a-\mathrm{Ca}_{2} \mathrm{Y}_{6.4} \mathrm{Eu}_{1.6} \mathrm{Si}_{6} \mathrm{O}_{26}$, $b-\mathrm{Ca}_{2} \mathrm{Gd}_{6.4} \mathrm{Eu}_{1.6} \mathrm{Si}_{6} \mathrm{O}_{26}, c-\mathrm{Ca}_{2} \mathrm{La}_{6.4} \mathrm{Eu}_{1.6} \mathrm{Ge}_{6} \mathrm{O}_{26}$.

На электронограмме образца (рис. 2, $b$ ) видны сильно размытые диффузные кольца (электронограммы были сняты с 6-ти разных участков образца), которые указывают на аморфный характер НП [20]. Значение ВЕТ поверхности нанопорошка $S_{\text {уд }}=232.25 \mathrm{~m}^{2} / \mathrm{g}$. Следовательно, средний размер частиц составляет $\sim 5.7 \mathrm{~nm}$.

Электронная микроскопия НП, полученного на основе $\mathrm{Ca}_{2} \mathrm{Gd}_{6.4} \mathrm{Eu}_{1.6} \mathrm{Si}_{6} \mathrm{O}_{26-\delta}$, показала, что наночастицы агломерированы. Агломераты в НП размером несколько сотен нанометров состоят из наночастиц неправильной формы с тенденцией к образованию квазисферических частиц. Наночастицы соединены между собой перешейками произвольной длины и формы. На (рис. $3, a$ ) показаны частицы нанообразца. TEM HR снимки показали, что НП состоит исключительно из аморфных частиц. На электронограмме НП (рис. $3, b$ ) также наблюдаются сильно размытые диффузные кольца, указывающие на аморфный характер образца (электронограммы сняты с 4-х разных участков образца). Значение ВЕТ поверхности нанопорошка $S_{\text {уд }}=172.63 \mathrm{~m}^{2} / \mathrm{g}$. Следовательно, средний размер частиц составляет $\sim 5.6 \mathrm{~nm}$.

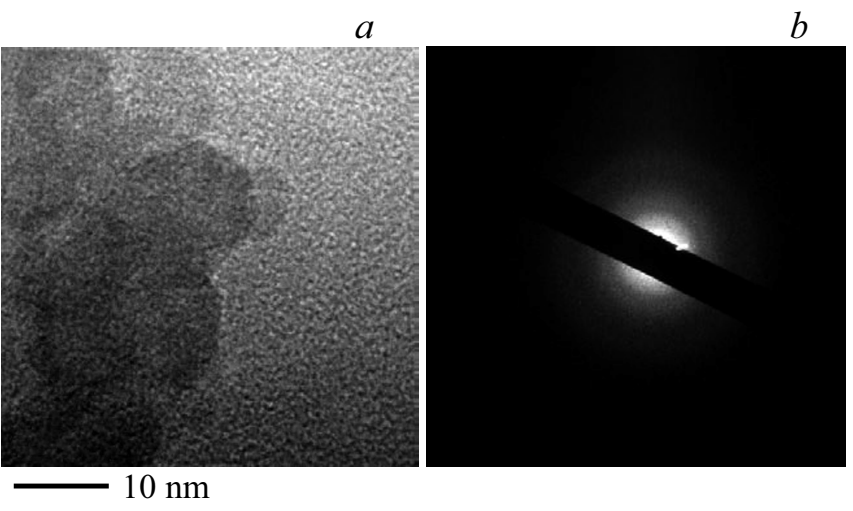

Pис. 2. TEM HR снимок (a) и электронограмма $(b)$ НП на основе $\mathrm{Ca}_{2} \mathrm{Y}_{6.4} \mathrm{Eu}_{1.6} \mathrm{Si}_{6} \mathrm{O}_{26}$.
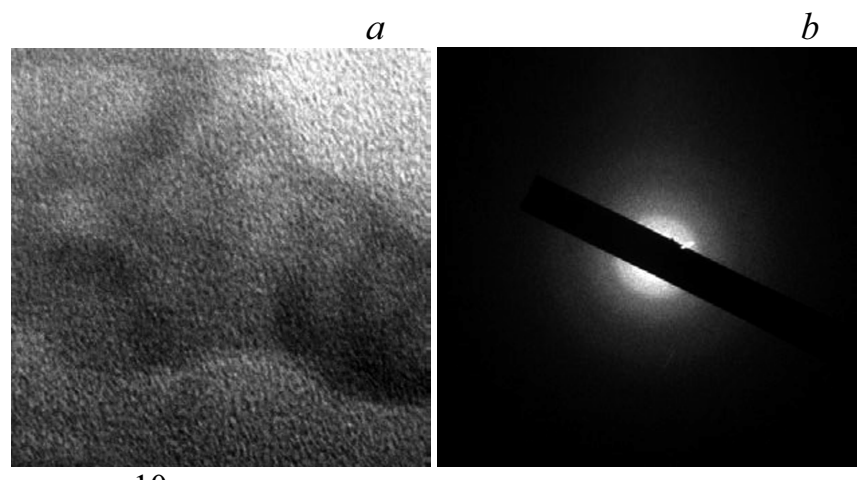

Pис. 3. TEM HR снимок (a) и электронограмма $(b)$ НП на основе $\mathrm{Ca}_{2} \mathrm{Gd}_{6.4} \mathrm{Eu}_{1.6} \mathrm{Si}_{6} \mathrm{O}_{26}$.

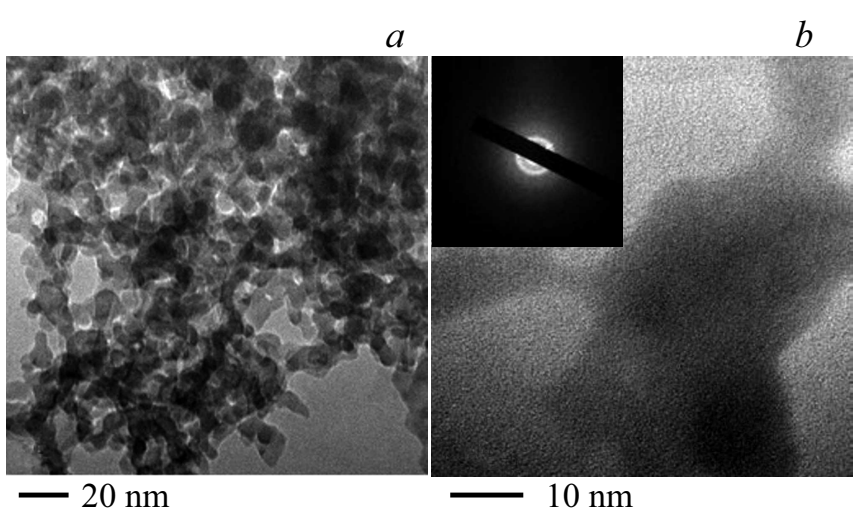

Pис. 4. TEM HR снимки $(a, b)$ и электронограмма (вставка) НП на основе $\mathrm{Ca}_{2} \mathrm{La}_{6.4} \mathrm{Eu}_{1.6}\left(\mathrm{GeO}_{4}\right)_{6} \mathrm{O}_{2}$. 


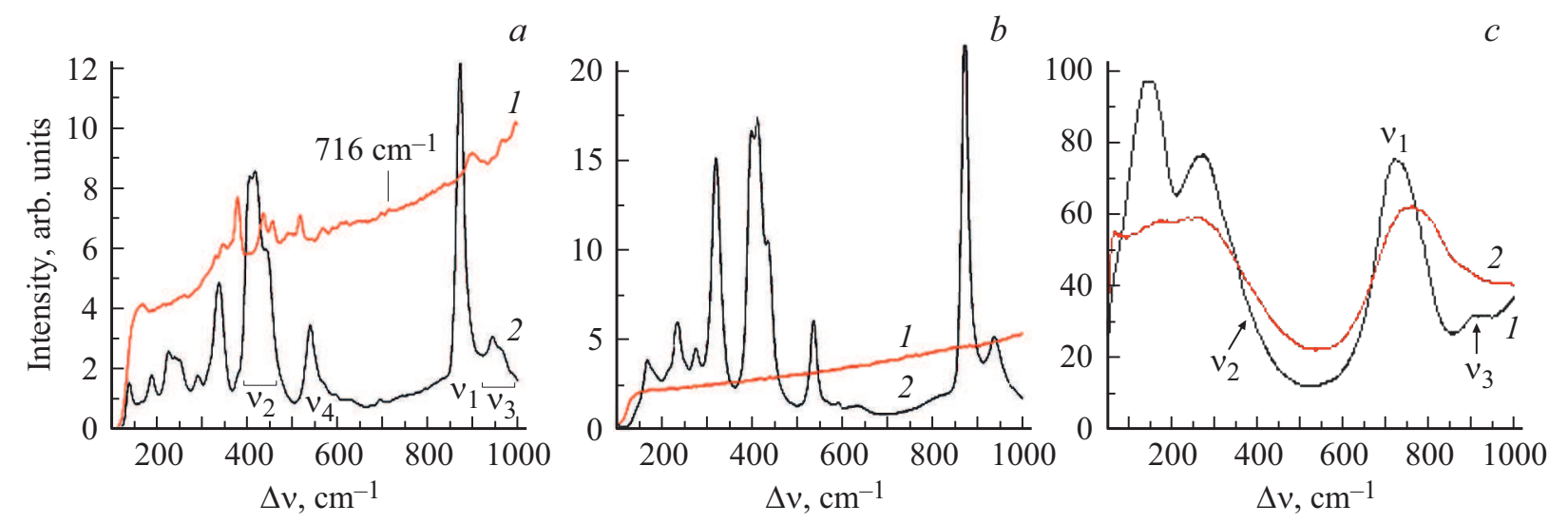

Рис. 5. КР-спектры ОП (1) и НП (2): $\left.\mathrm{Ca}_{2} \mathrm{Y}_{6.4} \mathrm{Eu}_{1.6}\left(\mathrm{SiO}_{4}\right)_{6} \mathrm{O}_{2}(a)\right), \mathrm{Ca}_{2} \mathrm{Gd}_{6.4} \mathrm{Eu}_{1.6}\left(\mathrm{SiO}_{4}\right)_{6} \mathrm{O}_{2}(b), \mathrm{Ca}_{2} \mathrm{La}_{6.4} \mathrm{Eu}_{1.6}\left(\mathrm{GeO}_{4}\right)_{6} \mathrm{O}_{2}(c)$.

Как и в предыдущих двух случаях микроскопия и электронография образца, полученного на основе $\mathrm{Ca}_{2} \mathrm{La}_{6.4} \mathrm{Eu}_{1.6}\left(\mathrm{GeO}_{4}\right)_{6} \mathrm{O}_{2-\delta}$, показывают, что наночастицы порошка сильно агломерированы и аморфны (рис. 4). Значение ВЕТ поверхности наночастиц составляет $S_{s p}=105.5 \mathrm{~m}^{2} / \mathrm{g}$. Следовательно, средний размер частиц составляет $\sim 9.9 \mathrm{~nm}$.

Рассмотрим КР-спектры объемных порошков (ОП) образцов $\mathrm{Ca}_{2} \mathrm{Y}_{6.4} \mathrm{Eu}_{1.6} \mathrm{Si}_{6} \mathrm{O}_{26-\delta}, \mathrm{Ca}_{2} \mathrm{Gd}_{6.4} \mathrm{Eu}_{1.6} \mathrm{Si}_{6} \mathrm{O}_{26-\delta}$, $\mathrm{Ca}_{2} \mathrm{La}_{6.4} \mathrm{Eu}_{1.6} \mathrm{Ge}_{6} \mathrm{O}_{26-\delta}$ и НП на их основе (рис. 5). Известно, что КР-спектры соединений, содержащих группы $\mathrm{SiO}_{4}$ с сильной ковалентной связью, позволяют получать информацию об изменении структуры в процессах плавления-кристаллизации [21]. Представляет интерес проследить за изменением КР-спектров силикатов в процессах испарения-отвердевания.

В кристаллах оксиапатита (пр. гр. $P 63 / m, Z=1$ ) site-симметрия атомов $\mathrm{Y}(\mathrm{Gd}, \mathrm{La})_{1}-4 f, \mathrm{Ca}-4 f$, $\mathrm{Y}(\mathrm{Gd}, \mathrm{La})_{2}-6 h, \mathrm{Si}(\mathrm{Ge})-6 h, \mathrm{O}-6 h, \mathrm{O}-12 i, \mathrm{O}-2 a$; $Z=1$. Европий, замещая атомы иттрия, гадолиния или лантана, может также занимать позиции $4 f$ и $6 h$. КР-спектр реальных кристаллов, содержащих примесь европия, а также НП, может отличаться от спектра идеальных кристаллов. Это отличие может быть обусловлено наличием структурных дефектов. Обозначены частоты $v_{1}, v_{2}, v_{3}$ и $v_{4}$ внутренних колебаний изолированных тетраэдров $\mathrm{SiO}_{4}$ и $\mathrm{GeO}_{4}$.

Спектр аморфного НП, полученного испарением $\mathrm{Ca}_{2} \mathrm{Y}_{6.4} \mathrm{Eu}_{1.6} \mathrm{Si}_{6} \mathrm{O}_{26-\delta}$ (рис. 5, a), показывает формирование структуры, несколько отличающейся от исходного поликристаллического образца. Расположение линий в спектре НП говорит о присутствии в нем в основном изолированных групп $\mathrm{SiO}_{4}$. В то же время появление в спектре частот в области $600-700 \mathrm{~cm}^{-1}$ свидетельствует в пользу формирования в образце незначительной концентрации полимеризованных кремнийкислородных фрагментов [3,22]. Появление новой частоты при $716 \mathrm{~cm}^{-1}$, относящейся, вероятно, к колебаниям мостиковых связей $\mathrm{Si}-\mathrm{O}-\mathrm{Si}$, является индикатором процессов полимеризации [21]. В процессе полимеризации, вероятно, участвует кислород $\mathrm{O}(4)$ в позиции $2 a$, не входящий в состав тетраэдров $\mathrm{SiO}_{4}$ [3].

Наблюдается сдвиг частоты $138 \mathrm{~cm}^{-1}$ в высокочастотную область и исчезновение частот внешних колебаний изолированных комплексов $\mathrm{SiO}_{4}$ (область $\left.187-250 \mathrm{~cm}^{-1}\right)$. Сдвиг линии при $337 \mathrm{~cm}^{-1}$ в высокочастотную область предположительно обусловлен увеличением величины силовой постоянной компоненты $E_{2 g}$ либрационного колебания комплекса $\mathrm{SiO}_{4}$. Частоты внутренних колебаний $v_{2}, v_{1}$ и $v_{3}$ также сдвигаются в высокочастотную область. Частота $v_{4}$, наоборот, сдвигается в низкочастотную область. Наблюдается относительное уменьшение интенсивностей линий при $v_{1}$ и $v_{2}$. Эти эффекты в спектре могут быть вызваны объединением в нанообразце большого числа кислородных вакансий, возникающих при испарении микрообразца и последующего его отвердевания. Объединение вакансий приводит к нарушению трансляционной симметрии. Такое нарушение симметрии может быть также причиной уменьшения интенсивности линии при $v_{1}[21]$.

Спектр аморфного НП, полученного испарением $\mathrm{Ca}_{2} \mathrm{Gd}_{6.4} \mathrm{Eu}_{1.6} \mathrm{Si}_{6} \mathrm{O}_{26-\delta}$ (рис. 5, $b$ ), имеет бесструктурную форму, отсутствуют характерные линии рассеяния. Это может быть также обусловлено нарушением трансляционной симметрии в НП и, следовательно, полным снятием запрета, который налагает закон сохранения квазиимпульса. Вследствие этого разрешены фононы с любыми волновыми векторами. [22]. В тоже время этот спектр очень похож на КР- спектр расплава $\mathrm{Ca}_{2} \mathrm{Gd}_{8} \mathrm{Si}_{6} \mathrm{O}_{26}$ [16]].

$\mathrm{B}$ спектрах образцов $\mathrm{Ca}_{2} \mathrm{La}_{6.4} \mathrm{Eu}_{1.6} \mathrm{Ge}_{6} \mathrm{O}_{26-\delta}$ и НП на его основе (рис. 5,c) отнесение линий проведено в соответствие с работой [23]. Линии частот внутренних колебаний тетраэдра $\mathrm{GeO}_{4}$ по сравнению с линиями КР тетраэдра $\mathrm{SiO}_{4}$ относительно широкие из-за большого числа кислородных вакансий вблизи $\mathrm{GeO}_{4}$ [23]. Область частот при $268 \mathrm{~cm}^{-1}$ можно отнести к колебаниям связи $\mathrm{La}-\mathrm{O}$ и трансляциям тетраэдров $\mathrm{GeO}_{4}$. Эта область частично перекрывается с линией частоты $v_{2}$. Полоса частот с максимумом при $144 \mathrm{~cm}^{-1}$ включает трансляции и либрационные колебания групп $\mathrm{GeO}_{4}$. При переходе 




Рис. 6. Спектры поглощения ОП (1) и НП (2): $\mathrm{Ca}_{2} \mathrm{Y}_{6.4} \mathrm{Eu}_{1.6}\left(\mathrm{SiO}_{4}\right)_{6} \mathrm{O}_{2}(a), \mathrm{Ca}_{2} \mathrm{Gd}_{6.4} \mathrm{Eu}_{1.6}\left(\mathrm{SiO}_{4}\right)_{6} \mathrm{O}_{2}(b), \mathrm{Ca}_{2} \mathrm{La}_{6.4} \mathrm{Eu}_{1.6}\left(\mathrm{GeO}_{4}\right)_{6} \mathrm{O}_{2}(c)$.

от поликристаллического образца к аморфному НП наблюдается сдвиг частот $v_{1}$ и $v_{2}$ в высокочастотную, а $\nu_{3}-$ в низкочастотную область. Частоты внешних колебаний сливаются в широкую полосу. Эти эффекты в спектре могут быть вызваны объединением в нанообразце большого числа кислородных вакансий, которые возникают при испарении поликристаллического образца [3] и большей степени разупорядочения структуры нанообразца по сравнению с ОП.

Рассмотрим изменение ширины запрещенной зоны $E_{g}$ образцов при переходе от ОП к НП. На рис. 6 представлены спектры поглощения образцов ОП и НП. Для $\mathrm{Ca}_{2} \mathrm{Y}_{8(1-x)} \mathrm{Eu}_{8 x}\left(\mathrm{SiO}_{4}\right)_{6} \mathrm{O}_{2}$ край поглощения расположен при $\sim 347.5 \mathrm{~nm}$, а для НП, полученного испарением этого силиката - при $294.5 \mathrm{~nm}$ (рис. 6, a). Следовательно, при переходе от ОП к НП величина $E_{g}$ увеличивается от $\sim 3.567$ до $\sim 4.209 \mathrm{eV}$. Край поглощения фосфоров обусловлен поглощением в полосе переноса заряда (состояние $4 f^{7} 2 p^{-1}$ ) аналогично $\mathrm{Sr}_{2} \mathrm{Gd}_{6.4} \mathrm{Eu}_{1.6}(\mathrm{SiO})_{6} \mathrm{O}_{2}$ и наноаморфному фосфору, полученному испарением ОП [3]. На рис. $6, b$ представлены спектры поглощения образцов $\mathrm{Ca}_{2} \mathrm{Gd}_{8(1-x)} \mathrm{Eu}_{8 x}\left(\mathrm{SiO}_{4}\right)_{6} \mathrm{O}_{2}$ и $\mathrm{HП}$ на основе этого люминофора. Для объемного фосфора край поглощения расположен при $\sim 365 \mathrm{~nm}$, что соответствует $E_{g}=3.396 \mathrm{eV}$. Полосу с максимумом при $\sim 256 \mathrm{~nm}$ можно отнести к переходу ${ }^{8} S \rightarrow{ }^{5} D$ иона $\mathrm{Gd}^{3+}[11]$. В области границы поглощения образца имеется протяженный экспоненциальный хвост, так называемый хвост Урбаха, характерный для аморфных сред [24]. Хвост Урбаха позволяет дать только оценку величины границы поглощения. Край поглощения расположен при $\sim 367 \mathrm{~nm}(\sim 3.34 \mathrm{eV})$.

Рассмотрим изменение ширины запрещенной зоны $E_{g}$ образцов германатов при переходе от ОП к НП. На рис. 6, в представлены спектры поглощения ОП $\mathrm{Ca}_{2} \mathrm{La}_{6.4} \mathrm{Eu}_{1.6} \mathrm{Ge}_{6} \mathrm{O}_{26}$ и НП на его основе. На спектре объемного образца имеются две полосы с максимумами при $\sim 246$ и $300 \mathrm{~nm}$. Эти полосы обусловлены переходом в состояние с переносом заряда для двух типов оптических центров, сформированных ионами $\mathrm{Eu}^{3+}$, находящихся в позициях $4 f$ и $6 h$. Для $\mathrm{Ca}_{2} \mathrm{La}_{6.4} \mathrm{Eu}_{1.6} \mathrm{Ge}_{6} \mathrm{O}_{26}$ край поглощения расположен при $\sim 343 \mathrm{~nm}$, что соответствует $E_{g}=3.61 \mathrm{eV}$. В области границы поглощения НП имеется протяженный экспоненциальный хвост (хвост Урбаха), характерный для аморфных сред, который не позволяет дать оценку величины $E_{g}$.

Уровень переноса заряда в $\mathrm{Eu}^{3+}$ формирует потолок запрещенной зоны. Оптическое поглощение при этом включает $p$-состоянии кислорода, чувствительное к изменению окружения иона $\mathrm{Eu}^{3+}$. Из спектров возбуждения ионов $\mathrm{Eu}^{3+}$ видно (рис. 7), что в объемных фосфорах максимум полосы возбуждения равен $270 \mathrm{~nm}$ $\left(\lambda_{e m}=614 \mathrm{~nm}\right)$. Для нанофосфоров этот максимум расположен при $\lambda_{\mathrm{ex}}<260 \mathrm{~nm}$ (рис. 8). Поэтому в объемных фосфорах уровень переноса заряда имеет меньшую энергию, чем в нанофосфорах. Край фундаментального поглощения в нанофосфорах должен быть расположен выше, чем в объемных фосфорах. Следовательно, наряду с различной стабильностью ближайших ионов кислорода $\mathrm{O}^{2-}$, окружающих ион $\mathrm{Eu}^{3+}$ в объемных и нанофосфорах, можно говорить о небольшом изменении этого окружения в нанофосфоре. Окружение ионов европия может измениться из-за незначительной конденсации тетраэдров $\mathrm{SiO}_{4}$.

На рис. 7, $a$ изображены спектры фотолюминесценции (ФЛ) люминофоров $\mathrm{Ca}_{2} \mathrm{Y}_{6.4} \mathrm{Eu}_{1.6} \mathrm{Si}_{6} \mathrm{O}_{26-\delta}$ и $\mathrm{Ca}_{2} \mathrm{Gd}_{6.4} \mathrm{Eu}_{1.6} \mathrm{Si}_{6} \mathrm{O}_{26-\delta}$. Наряду с узкими линиями свечения ионов $\mathrm{Eu}^{3+}$ имеются широкие полосы $\left(\lambda_{\max }=443\right.$ нм$)$, обусловленные свечением ионов $\mathrm{Eu}^{2+}$ (переход $4 f^{6} 5 d \rightarrow 4 f^{7}\left({ }^{8} S_{7 / 2}\right)$ ). Широкие полосы имеют слабую структуру, которая, вероятно, обусловлена взаимодействием $4 f^{6}$ - и $5 d$-электронов [25]. Поскольку ион $\mathrm{Eu}^{3+}$ при замещении $\mathrm{Y}(\mathrm{Gd})$ занимает в структуре силикатов две кристаллографические позиции $4 f$ и $6 h$, то $\mathrm{Eu}^{3+}$ формирует два типа оптических центров. Спектры является суммарными спектрами люминесценции этих двух центров. 


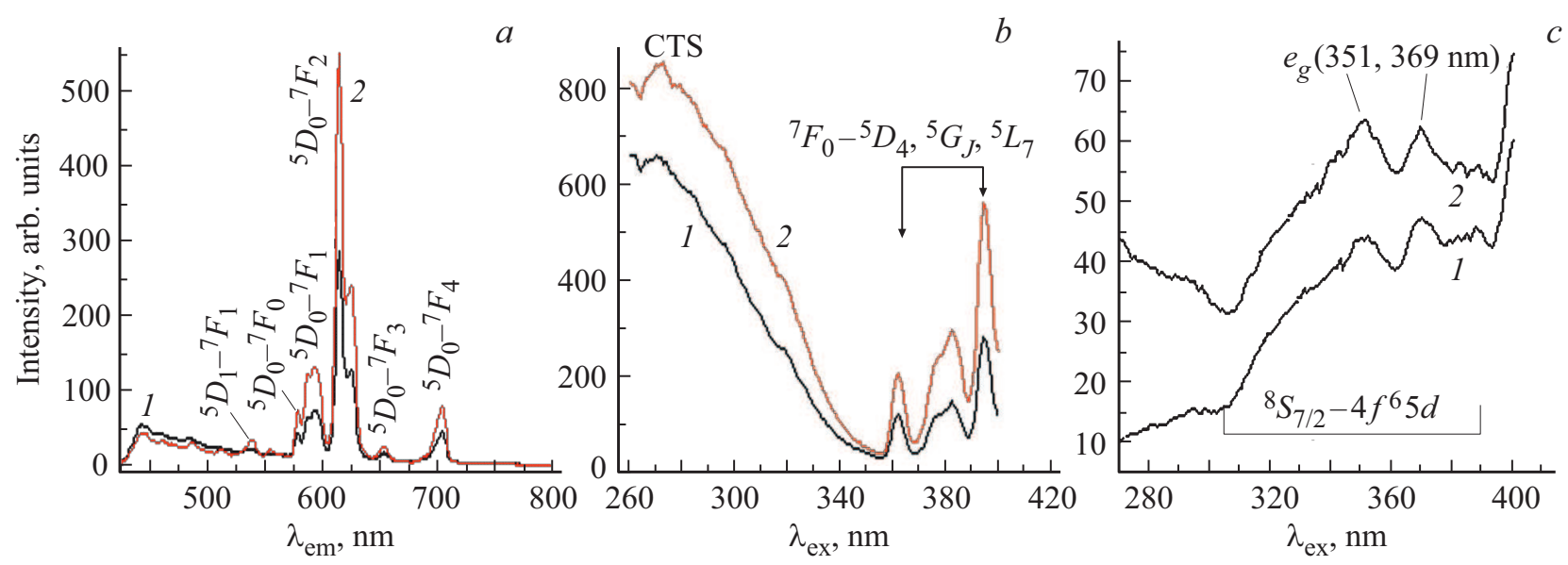

Рис. 7. Спектры ФЛ и ВФЛ $\mathrm{Ca}_{2} \mathrm{Y}_{6.4} \mathrm{Eu}_{1.6} \mathrm{Si}_{6} \mathrm{O}_{26-\delta}(1)$ и $\mathrm{Ca}_{2} \mathrm{Gd}_{6.4} \mathrm{Eu}_{1.6} \mathrm{Si}_{6} \mathrm{O}_{26-\delta}$ (2): спектры ФЛ образцов 1,2 (a); спектры ВФЛ $\left(\lambda_{\mathrm{em}}=614 \mathrm{~nm}\right)(b)$ и $\left(\lambda_{\mathrm{em}}=443 \mathrm{~nm}\right)$ образцов $1,2(c)$.

Механизм образования ионов $\mathrm{Eu}^{2+}$ может быть, по-нашему мнению, аналогичен изложенному в работах [3,26-28]. При синтезе поликристаллов $\mathrm{Ca}_{2} \mathrm{Y}(\mathrm{Gd})_{6.4} \mathrm{Eu}_{1.6} \mathrm{Si}_{6} \mathrm{O}_{26-\delta} \quad$ в процессе высокотемпературного обжига смеси исходных компонентов в формируемом оксиапатите в позиции $4 f$ происходит образование вакансий $V_{\mathrm{Ca}}^{\|}$. Вакансии $V_{\mathrm{Ca}}^{\|}$передают свой отрицательный заряд двум ионам $\mathrm{Eu}_{\mathrm{Y}}^{3+}$ или $\mathrm{Eu}_{\mathrm{Gd}}^{3+}$. Это приводит к образованию $\mathrm{Eu}^{2+}$. Поэтому химическую формулу образцов следует записывать в виде $\mathrm{Ca}_{2} \mathrm{Y}(\mathrm{Gd})_{6.4} \mathrm{Eu}_{1.6} \mathrm{Si}_{6} \mathrm{O}_{26-\delta}$, где $\delta-$ кислородная нестехиометрия. В структуре апатита ионы $\mathrm{Eu}^{2+}$ формируют также два типа оптических центров в позициях $6 h$ и $4 f$ [26]. Спектры свечения этих двух центров является суммарными спектрами, образуя указанные широкие полосы.

В спектрах возбуждения (ВФЛ) объемных фосфоров (рис. $7, b$ ) для $\lambda_{\mathrm{em}}=614 \mathrm{~nm}$ имеются полосы СТS и линии, обусловленные переходами ${ }^{7} F_{0} \rightarrow{ }^{5} D_{4},{ }^{5} G_{2}$, ${ }^{5} L_{7}$. Для $\lambda_{\mathrm{em}}=443 \mathrm{~nm}$ спектры ВФЛ $\left(\mathrm{Eu}^{2+}\right)$ различаются в интервале длин волн 260-305 nm (рис. 7,c). Для люминофора $\mathrm{Ca}_{2} \mathrm{Gd}_{6.4} \mathrm{Eu}_{1.6} \mathrm{Si}_{6} \mathrm{O}_{26-\delta}$ в указанном интервале длин волн наблюдается увеличение интенсивности поглощения в отличие от люминофора $\mathrm{Ca}_{2} \mathrm{Y}_{6.4} \mathrm{Eu}_{1.6} \mathrm{Si}_{6} \mathrm{O}_{26-\delta}$. Широкие полосы в интервале $305-390 \mathrm{~nm}$ обусловлены переходом с уровня $4 f^{7}\left({ }^{8} S_{7 / 2}\right)$ на $e_{g}$-уровень $5 d$-состояния ионов $\mathrm{Eu}^{2+}$. На указанных широких полосах для обоих люминофоров имеются два максимума при 351 и $369 \mathrm{~nm}$, обусловленные, вероятно, снятием вырождения уровня $e_{g}$. Это указывает на достаточно сильное взаимодействие между $4 f^{6}$-электронами и $5 d$-электроном.

На рис. 8 изображены спектры ФЛ и ВФЛ нанофосфоров, полученных испарением образцов $\mathrm{Ca}_{2} \mathrm{Y}_{6.4} \mathrm{Eu}_{1.6} \mathrm{Si}_{6} \mathrm{O}_{26-\delta} \quad$ и $\quad \mathrm{Ca}_{2} \mathrm{Gd}_{6.4} \mathrm{Eu}_{1.6} \mathrm{Si}_{6} \mathrm{O}_{26-\delta}$. Спектры ФЛ представляют собой широкие полосы, обусловленные свечением ионов $\mathrm{Eu}^{2+}$. Излучение ионов $\mathrm{Eu}^{3+}$ почти потушено (рис. $8, a-c$ ). Заметная интенсивность люминесценции ионов $\mathrm{Eu}^{3+}$ наблюдается только для переходов ${ }^{5} D_{0} \rightarrow{ }^{7} F_{1,2}$. Спектр, изображенный на рис. $8, b$, записан для нанолюминофора, полученного ИЭП образца $\mathrm{Ca}_{2} \mathrm{Y}_{6.4} \mathrm{Eu}_{1.6} \mathrm{Si}_{6} \mathrm{O}_{26-\delta}$ в атмосфере аргона. Интенсивность переходов ${ }^{5} D_{0} \rightarrow{ }^{7} F_{1,2}$ в этом спектре значительно меньше, чем для аналогичного образца, но полученного испарением в вакууме (рис. 8, a), а интенсивность свечение ионов $\mathrm{Eu}^{2+}$ больше. Можно предположить, что дополнительно образуются ионы $\mathrm{Eu}^{2+}$ в нанообразцах за счет радиационного восстановления $\mathrm{Eu}^{3+} \rightarrow \mathrm{Eu}^{2+}$. Подобная редукция при воздействии ионизирующего излучения $(\alpha, \beta, \gamma$, рентгеновское и лазерное излучение) возможна в соединениях, содержащих группы $\mathrm{MO}_{4}$ $(M=\mathrm{Si}, \mathrm{B}, \mathrm{Al}, \mathrm{P})$ [29,30]. Механизм этого процесса может быть следующий. Электрон высвобождается из тетраэдра $\mathrm{SiO}_{4}^{4-}$ за счет разрыва связи $\mathrm{Si}-\mathrm{O}$ в процессе ИЭП. Возникает состояние $\left(\mathrm{SiO}_{4}^{3-}\right)^{+}[31]$. Освобожденный электрон захватывается ионами $\mathrm{Eu}^{3+}$, являющимися электронными ловушками [32], $\mathrm{Eu}^{3+}$ восстанавливается до $\mathrm{Eu}^{2+}$. Следовательно, возможны следующие реакции:

$$
\begin{gathered}
\mathrm{SiO}_{4}^{4-} \stackrel{\text { ИЭП }}{\longrightarrow}\left(\mathrm{SiO}_{4}^{3-}\right)^{+}+e, \\
\mathrm{Eu}^{3+}+e \rightarrow \mathrm{Eu}^{2+} .
\end{gathered}
$$

Для нанофосфора, полученного на основе $\mathrm{Ca}_{2} \mathrm{Gd}_{6.4} \mathrm{Eu}_{1.6} \mathrm{Si}_{6} \mathrm{O}_{26-\delta}$, интенсивность переходов ${ }^{5} D_{0} \rightarrow{ }^{7} F_{1,2}$ также низкая. В тоже время интенсивность свечения ионов $\mathrm{Eu}^{2+}$ увеличивается в нанофосфоре по сравнению с интенсивность ионов $\mathrm{Eu}^{2+}$ в объемном образце. Следовательно, в этом нанообразце также происходит восстановление ионов $\mathrm{Eu}^{3+} \rightarrow \mathrm{Eu}^{2+}$.

Интенсивность свечения ионов $\mathrm{Eu}^{2+}$ почти одинакова для $\mathrm{Ca}_{2} \mathrm{Y}_{6.4} \mathrm{Eu}_{1.6} \mathrm{Si}_{6} \mathrm{O}_{26-\delta}$ и нанолюминофора, полученного на его основе в вакууме (рис. 7, $a$ и 8, $a$ ). Свечение ионов $\mathrm{Eu}^{3+}$ в нанообразце значительно потушено. Вероятно, в образце восстановление $\mathrm{Eu}^{3+} \rightarrow \mathrm{Eu}^{2+}$ почти 



Рис. 8. Спектры ФЛ и ВФЛ нанофосфоров, полученных на основе $\mathrm{Ca}_{2} \mathrm{Y}_{6.4} \mathrm{Eu}_{1.6} \mathrm{Si}_{6} \mathrm{O}_{26-\delta}(1)$ и $\mathrm{Ca}_{2} \mathrm{Gd}_{6.4} \mathrm{Eu}_{1.6} \mathrm{Si}_{6} \mathrm{O}_{26-\delta}$ (2): спектры ФЛ образцов $1,2(a-c)$; спектры ВФЛ $\left(\lambda_{\mathrm{em}}=614 \mathrm{~nm}\right)(d)$ и $\left(\lambda_{\mathrm{em}}=443 \mathrm{~nm}\right)$ образцов $1,2(e)$.

не происходит. Малый выход ФЛ ионов $\mathrm{Eu}^{3+}$ можно объяснить с помощью конфигурационной диаграммы в модели Декстера-Клика-Рассела [33]. Таким образом, восстановление ионов $\mathrm{Eu}^{3+} \rightarrow \mathrm{Eu}^{2+}$ зависит не только от атмосферы, в которой происходит испарение, но и от состава исходных объемных образцов.

Сравнивая соответствующие величины $\lambda_{\max }$ можно заметить, что при переходе ОП $\rightarrow$ НП наблюдается небольшой красный сдвиг $\lambda_{\max }$. Этот сдвиг можно объяснить увеличением ковалентности связи $\mathrm{Eu}^{2+}-\mathrm{O}$ в оптических центрах нанообразцов [34].

Рассмотрим спектры ВФЛ нанофосфоров. В этих (рис. $8, d$ ) для $\lambda_{\mathrm{em}}=614 \mathrm{~nm}$ линии, обусловленные переходами ${ }^{7} F_{0} \rightarrow{ }^{5} D_{4},{ }^{5} G_{J},{ }^{5} L_{7}$, имеют значительно меньшую интенсивность по сравнению со спектрами ВФЛ объемных фосфоров (рис. 7, $b$ ). Это подтверждает относительно небольшое число ионов $\mathrm{Eu}^{3+}$, участвующих в процессе люминесценции.

Для нанообразца на основе $\mathrm{Ca}_{2} \mathrm{Y}_{6.4} \mathrm{Eu}_{1.6} \mathrm{Si}_{6} \mathrm{O}_{26-\delta}$ спектр ВФЛ $\mathrm{Eu}^{2+}\left(\lambda_{\mathrm{em}}=443 \mathrm{~nm}\right)$ почти такой же, как для объемного образца. Для нанообразца на основе $\mathrm{Ca}_{2} \mathrm{Gd}_{6.4} \mathrm{Eu}_{1.6} \mathrm{Si}_{6} \mathrm{O}_{26-\delta}$ уровень ${ }^{2} e_{g}$ становится вырожденным и возникают дополнительные полосы возбуждения в области 260-320 nm (рис. 8,e). Эти полосы, вероятно, соответствуют переходам ${ }^{8} S_{7 / 2} \rightarrow{ }^{6} P_{J}$ иона
$\mathrm{Eu}^{2+}$. Следовательно, при переходе ОП $\rightarrow$ НП поле лигандов вокруг $\mathrm{Eu}^{2+}$ изменяется. Это может быть обусловлено (как сказано выше) нарушением трансляционной симметрии в НП. Ослабляется связь $4 f$ и $5 d$-электронов. Возникает вырождение уровня ${ }^{2} e_{g}$. Ион $\mathrm{Eu}^{2+}$ из состояния ${ }^{6} P_{J}$ безызлучательно релаксирует в состояние ${ }^{2} e_{g}$, из которого и происходит люминесценция. Для нанообразца на основе $\mathrm{Ca}_{2} \mathrm{Y}_{6.4} \mathrm{Eu}_{1.6} \mathrm{Si}_{6} \mathrm{O}_{26-\delta}$ поле лигандов вокруг $\mathrm{Eu}^{2+}$ почти не изменяется.

На рис. 9, $a, b$ представлены спектры ФЛ и ВФЛ люминофора $\mathrm{Ca}_{2} \mathrm{La}_{6.4} \mathrm{Eu}_{1.6} \mathrm{Ge}_{6} \mathrm{O}_{26-\delta}$. Ионы $\mathrm{Eu}^{3+}$ образуют два типа оптических центров при $\lambda_{\max }=577.4$ и $578 \mathrm{~nm}$. Для нанообразца на основе $\mathrm{Ca}_{2} \mathrm{La}_{6.4} \mathrm{Eu}_{1.6} \mathrm{Ge}_{6} \mathrm{O}_{26-\delta}$ спектр ФЛ существенно изменяется (рис. 9, $c$ ). Возникает свечение в интервале $450-750 \mathrm{~nm}\left(\lambda_{\mathrm{ex}}=394 \mathrm{~nm}\right)$, обусловленное ионами $\mathrm{Eu}^{2+}$. Механизм образования ионов $\mathrm{Eu}^{2+}$, понашему мнению, аналогичен таковому для нанофосфоров на основе силикатных образцов (реакции 1,2). Радиационное восстановление $\mathrm{Eu}^{3+} \rightarrow \mathrm{Eu}^{2+}$ согласно реакциям (3) и (4)

$$
\begin{gathered}
\mathrm{GeO}_{4}^{4-} \stackrel{\text { иэП }}{\longrightarrow}\left(\mathrm{GeO}_{4}^{3-}\right)^{+}+e, \\
\mathrm{Eu}^{3+}+e \rightarrow \mathrm{Eu}^{2+} .
\end{gathered}
$$

Сравнивая рис. $8, a-c$ и рис. $9, c$, можно заключить, что в НП, полученном испарением германата, степень вос- 

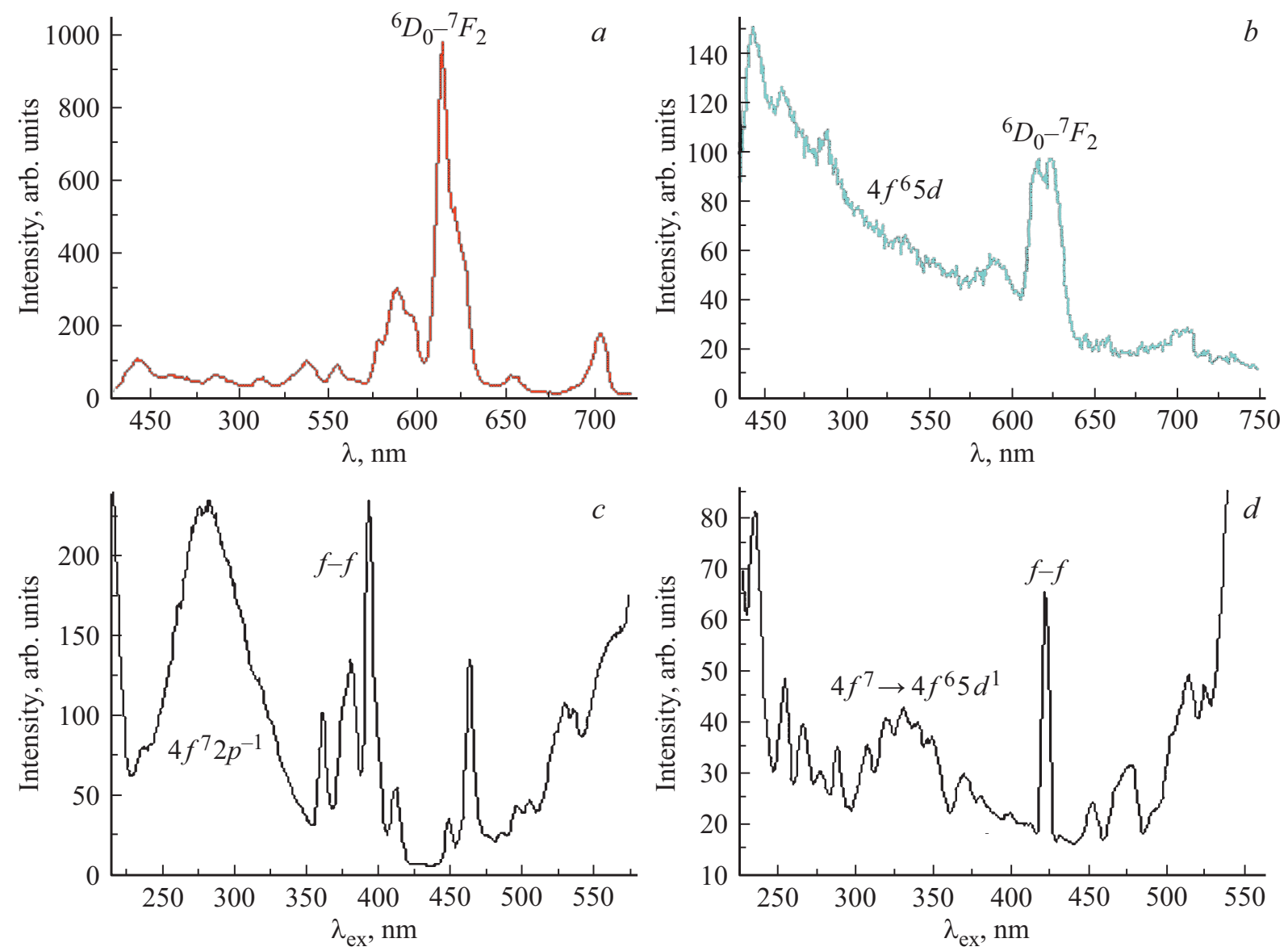

Рис. 9. Спектры ФЛ $\mathrm{Ca}_{2} \mathrm{La}_{6.4} \mathrm{Eu}_{1.6} \mathrm{Ge}_{6} \mathrm{O}_{26-\delta} \quad(a)$, НП (b) НП $\left(\lambda_{\mathrm{em}}=443 \mathrm{~nm}\right)(d)$.

становления ионов $\mathrm{Eu}^{3+}$ до $\mathrm{Eu}^{2+}$ относительно меньше, чем в НП, полученном при ИЭП силикатов. Электрон высвобождается из тетраэдра $\mathrm{GeO}_{4}^{4-}$ за счет разрыва связи $\mathrm{Ge}-\mathrm{O}$ в процессе ИЭП и захватывается электронными ловушками (ионы $\mathrm{Eu}^{3+}$ ). Можно предположить, что различие в степени восстановления ионов $\mathrm{Eu}^{3+}$ обусловлено различием высвобождения электронов при разрыве связей $\mathrm{Si}-\mathrm{O}$ и $\mathrm{Ge}-\mathrm{O}$, а также характеристиками электронных ловушек в силикатных и германатных нанообразцах. В результате этого цвет свечения нанолюминофоров, полученных на основе силикатов становится синим, а полученного на основе германата - белым.

Спектр свечения ионов $\mathrm{Eu}^{2+}$, изображенный на рис. 9, $c$, разложен на две компоненты Гаусса (на рис. 9, не изображены) с $\lambda_{\max }=455.4$ и $582.2 \mathrm{~nm}$. Следовательно, ион $\mathrm{Eu}^{2+}$ образует два типа оптических центров, вероятно, в позициях $4 f$ и $6 h$. На рис. $9, d$ изображен спектр ВФЛ нанолюминофора $\left(\lambda_{\mathrm{em}}=443 \mathrm{~nm}\right)$. В спектре наблюдаются широкая полоса (переход $4 f^{7}-4 f^{6} 5 d^{1}$ ионов $\left.\mathrm{Eu}^{2+}\right)$ и линии, соответствующие $f-f$ - переходам ионов $\mathrm{Eu}^{3+}$. Вид спектра возбуждения указывает на резонансное взаимодействие ионов $\mathrm{Eu}^{2+}$ и $\mathrm{Eu}^{3+}$.

На спектр ФЛ $\mathrm{Eu}^{2+}$ накладывается излучение ионов $\mathrm{Eu}^{3+}$. Для перехода ${ }^{5} D_{0}-{ }^{7} F_{2}$ наиболее интенсивные линии наблюдаются при 615 и $623 \mathrm{~nm}$. Для объемного образца линии этого перехода наблюдаются при 613 и $620 \mathrm{~nm}$. Известно, что электрический дипольный переход ${ }^{5} D_{0} \rightarrow{ }^{7} F_{2}$ является сверхчувствительным к Siteсимметрии иона $\mathrm{Eu}^{3+}$ в оптическом центре. Сдвиг линий перехода в НП в сторону большей энергии и перераспределение их относительных интенсивностей указывает на некоторое искажение локального окружения ионов $\mathrm{Eu}^{3+}$ в нанофосфоре по сравнению с ОП, вероятно, из-за объединения в нанообразце большого числа кислородных вакансий, которые возникают в процессе ИЭП. Наложение излучения ионов $\mathrm{Eu}^{2+}$ и $\mathrm{Eu}^{3+}$ дает белое свечение люминофора с цветовыми координатами $x=0.313, y=0.320$.

\section{4. Заключение}

Разработан способ получения новых сложнооксидных наноалюминофоров. Получены новые наноаморфные люминофоры путем испарения электронным пучком в вакууме или атмосфере аргона красно-оранжевых кристаллофосфоров составов $\mathrm{Ca}_{2} M_{8}\left(\mathrm{SiO}_{4}\right)_{6} \mathrm{O}_{2}: \mathrm{Eu}(M=\mathrm{Y}$, $\mathrm{Gd})$ и $\mathrm{Ca}_{2} \mathrm{La}_{8}\left(\mathrm{GeO}_{4}\right)_{6} \mathrm{O}_{2}: \mathrm{Eu}$. Предположительно обнаружено восстановление ионов $\mathrm{Eu}^{3+} \rightarrow \mathrm{Eu}^{2+}$ в электронном 
пучке за счет разрыва связи $\mathrm{Si}(\mathrm{Ge})-\mathrm{O}$ в тетраэдрах $\mathrm{SiO}_{4}$ и $\mathrm{GeO}_{4}$ в процессе испарения образцов и захвата высвободившегося электрона ионами $\mathrm{Eu}^{3+}$. Изучены спектрально-люминесцентные характеристики поликристаллических фосфоров $\mathrm{Ca}_{2} M_{8}\left(\mathrm{SiO}_{4}\right)_{6} \mathrm{O}_{2}: \mathrm{Eu} \quad(M=\mathrm{Y}$, $\mathrm{Gd}), \mathrm{Ca}_{2} \mathrm{La}_{8}\left(\mathrm{GeO}_{4}\right)_{6} \mathrm{O}_{2}: \mathrm{Eu}$ и полученных на их основе наноаморфных фосфоров. Установлено, что при переходе от объемного образца $\mathrm{Ca}_{2} \mathrm{Y}_{8(1-x)} \mathrm{Eu}_{8 x}\left(\mathrm{SiO}_{4}\right)_{6} \mathrm{O}_{2}$ к НП величина $E_{g}$ увеличивается. Обнаружена модификация КР-спектров при уменьшении частиц образцов от объемного до наноразмерного состояния. Полученные нанофосфоры при ИЭП силикатов РЗЭ имеют синее свечение, а на основе германата - белое.

\section{Список литературы}

[1] S.Y. Raghvendra, K.D. Ranu, M. Kumar, A.C. Pande. J. Lumin. 129, 1078 (2009).

[2] R.N. Bhargava, V. Chhabra, B. Kulkarni, J.V. Veliadis. Phys. Status Solidi b 210, 621 (1998).

[3] M.G. Zuev, S.Yu. Sokovnin, V.G. Il'ves, I.V. Baklanova, A.A. Vasin. J. Solid State Chem. 218, 164 (2014).

[4] C. Li, A. Lagriffoul, R. Moncorge, J.C. Souriau, C. Borel, Ch. Wyon. J. Lumin. 62, 157 (1994).

[5] M.D. Chambers, P.A. Rousseve, D.R. Clarke. J. Lumin. 129, 263 (2009).

[6] N. Xiumei, L. Jun, L. Zhe, Q. Xiwei, L. Mingua, W. Xiaoqiang. J. Rare Earths 26, 904 (2008).

[7] G.S. Rama Raju, H.C. Jung, J.Y. Park, B.K. Moon, R. Balakrishnaiah, J.H. Jeong, J.H. Kim. Sensors Actuators B 146, 395 (2010).

[8] Meidan Que, Zhipeng Ci, Yuhua Wang, Ge Zhu, Yurong Shi, Shuangyu Xin. J. Lumin. 144, 64 (2013).

[9] S. Qi, Y. Huang, T. Tsuboi, W. Huang, H.J. Seo. Opt. Mater. Express 4, 396 (2014)

[10] J.K. Han, M.E. Hannah, A. Piquette, J. Micone, G.A. Hirata, J.B. Talbot, K.C. Mishra, J. McKittrick. J. Lumin. 133, 184 (2013).

[11] B. Chu, C. Guo, Q. Su. Materials Chem. Phys. 84, 279 (2004).

[12] C. Peng, G. Li, Z. Hou, M. Shang, J. Lin. Mater. Chem. Phys. 136, 1008 (2012).

[13] K.-Y. Yeh, C.-C. Yang, W.-R. Liu, M.G. Brik. Opt. Mater. Express 6, 418 (2016).

[14] Yu-Chun Li, Yen-Hwei Chang, Bin-Siang Tsai, YuChung Chen, Yu-Feng Lin. J. Alloys Comp. 416, 199 (2006).

[15] S.Yu. Sokovnin, V.G. Il'ves, M.G. Zuev. Production of complex metal oxide nanopouders using pulsed electron beam in low-pressure gas for biomaieriats application. Ch. 2. In: Engineering of Nanobiomaterials Applications of Nanobiomaterials. V. 2. / Ed. A. Grumezescu. Elsevier (2016).

[16] S. Thomas. Silicate and Aluminate Based Dielectric Ceramics for Microwave Communication / Under the guidance and supervision of Dr. M.T. Sebastian (Supervisor). National Institute for Interdisciplinary Science and Technology (CSIR), Thiruvananthapuram (2010).

[17] С. Брунауэр. Адсорбция газов и паров. Физическая адсорбция. ГИИЛ, М. (1948). Т. 1. 784 с.
[18] Powder Diffraction File ICDD PDF-4 PDF2007 card 00-0290320 Smith, McCarthy.

Penn State University, University Park, (1976) Pennsylvania, USA, ICDD Grant-in-Aid.

[19] Landolt-Börnstein. Group III Condensed Matter Vol. 7G / Eds: K.-H. Hellwege, A.M. Hellwege. Springer-Verlag, Berlin Heidelberg (1974).

[20] L.-M. Peng, S.L. Dudarev, M.J. Whelan. High Energy Electron Diffraction and Microscopy. Oxford University Press (2004).

[21] Ю.К. Воронько, А.А. Соболь, В.Е. Шукшин, А.И. Загуменный, Ю.Д. Заварцев, С.А. Кутовой. ФТТ 54, 8, 1533 (2012).

[22] А.Н. Лазарев, А.П. Миргородский, И.С. Игнатьев. Колебательные спектры сложных окислов. Наука, Л. (1975). $296 \mathrm{c.}$

[23] Evelyn Rodríguez-Reyna, Antonio F. Fuentes, Miroslaw Maczka, Jerzy Hanuza, Khalid Boulahya, Ulises Amador. Solid State Sci. 8, 168 (2006).

[24] И.А. Вайнштейн, А.Ф. Зацепин, В.С. Кортов, Ю.В. Щапова. ФТT 42, 2, 224 (2000).

[25] F.M. Ryan, W. Lehmann, D.W. Feldman, J. Murphy, J. Electrochem. Sac.: Solid-State Sci. Technol. 121, 1475 (I974).

[26] M.G. Zuev, A.M. Karpov, A.S. Shkvarin. J. Solid State Chem. 184, 52 (2011).

[27] Cuimiao Zhang, Jun Yang, Cuikun Lin, Chunxia Li, Jun Lin. J. Solid State Chem. 182, 1673 (2009).

[28] Jian Chen, Yan-gai Liu, Haikun Liu, Dexin Yang, Hao Ding, Minghao Fang, Zhaohui Huang. Cite this: RSC Adv. 4, 18234 (2014).

[29] Manveer Singh, P.D. Sahare, Pratik Kumar, Shaila Bahl. Columbia International Publishing. J. Lumin. Appl. 3, 1 (2016).

[30] E. Malchukova, B. Boizot. Mater. Res. Bull. 45, 1299 (2010).

[31] А.Ф. Зацепин, А.И. Кухаренко, В.А. Пустоваров, В.Ю. Яковлев, С.О. Чолах. ФТТ 51, 3, 437 (2009).

[32] Ryosuke Yokota. J. Phys. Soc. Jpn. 23129 (1967).

[33] G. Blasse, A. Bril. Philips Techn. Rev. 31, 304 (1970).

[34] P. Dorenbos. J. Lumin. 104, 239 (2003).

Редактор Д.В. Жуманов 